\title{
ADP-Ribosylation: Activation, Recognition, and Removal
}

\author{
Nan Li, and Junjie Chen*
}

\begin{abstract}
ADP-ribosylation is a type of posttranslational modification catalyzed by members of the poly(ADP-ribose) (PAR) polymerase superfamily. ADP-ribosylation is initiated by PARPs, recognized by PAR binding proteins, and removed by PARG and other ADP-ribose hydrolases. These three groups of proteins work together to regulate the cellular and molecular response of PAR signaling, which is critical for a wide range of cellular and physiological functions.
\end{abstract}

\section{INTRODUCTION}

ADP-ribosylation is a type of protein posttranslational modification initiated by a group of enzymes named poly(ADP-ribose) (PAR) polymerases (PARPs). The PARPs can hydrolyze nicotinamide adenine dinucleotide and transfer ADP-ribose to substrates. There are two types of ADP-ribosylation: mono-ADPribosylation is a modification in which only one unit of ADP. ribose is transferred to Glu/Asp/Lys residues of target proteins, while poly-ADP-ribosylation (PARsylation) involves several units of ADP-ribose being transferred to specific residues of target proteins to form ADP-ribose polymers (Hassa and Hottiger, 2008; Schreiber et al., 2006).

Posttranslational modification by PARsylation regulates cellular functions via several mechanisms. For example, ribosylation of some DNA repair proteins is required for their recruitment to DNA damage sites; ribosylation of substrate proteins can also affect protein-protein interactions, especially in the case of PARbinding proteins that specifically recognize the ribosylated target proteins; ribosylation of target proteins can also facilitate the recruitment of E3 ubiquitin ligase, which promotes target protein degradation via proteasomal pathway. Through these distinct mechanisms, PARsylation participates in a wide range of cellular processes, including DNA damage response, transcription, chromatin remodeling, telomere protection, cell proliferation, cell death, and tumorigenesis. In this review, we summarize our current understanding of PARP family members and their func-

Department of Experimental Radiation Oncology, Unit 66, The University of Texas MD Anderson Cancer Center, Houston, TX 77030, USA *Correspondence: jchen8@mdanderson.org

Received 30 August, 2013; accepted 2 September, 2013; published online 27 January, 2014

Keywords: ADP-Ribosylation, PARG, PARP, PAR recognition domain tions in various aspects of cellular processes. In addition, we categorize the PAR recognition domains and describe the importance of PAR-binding motifs in signal transduction. Moreover, we introduce the unique functions of several enzymes that are specifically involved in the removal of ADP-ribosylation, which indicates the reversible nature of ADP-ribo-sylation as a signaling moiety.

\section{THE PARP FAMILY MEMBERS AND THEIR DIVERSE CELLULAR FUNCTIONS}

The PARP family has 17 members, which can be categorized into five subgroups according to their domains or functions (Fig. 1): DNA damage-dependent PARPs (PARP1, PARP2, and PARP3), tankyrases (tankyrase1/PARP5a and tankyrase2/ PARP5b), CCCH-type PARPs (PARP7, PARP12, and PARP13), macro-PARPs [B-aggressive lymphoma 1 (BAL1)/PARP9, BAL2/ PARP14, and BAL3/PARP15], and other PARPs (PARP4, PARP6, PARP8, PARP10, PARP11, and PARP16) (Gibson and Kraus, 2012).

\section{DNA damage-dependent PARPs}

The DNA damage-dependent PARPs are normally activated by DNA breaks. PARP1, the first identified and the best understood PARP, is a nuclear protein and has three known functional domains: the DNA-binding domain at its very $\mathrm{N}$-terminal region (Zinc finger domain); the auto-modification domain in the middle (i.e. BRCT domain), which is also where PARP1 interacts with its substrates as well as the region that undergoes automodification/poly-ribosylation; and the C-terminal catalytic domain, which binds to nicotinamide adenine dinucleotide and promotes the formation of ADP-ribose chain on its substrates as well as itself (Rouleau et al., 2010). PARP1 is required for DNA single-strand break repair (SSBR) and base-excision repair (BER) by binding to and recruiting XRCC1 to DNA damage site (Parsons et al., 2005). In addition, PARP1 also interacts with DNA double-strand break repair proteins such as $\mathrm{Ku}$ and DNA-PKcs and participates in NHEJ (nonhomologous end joining) repair pathway (Kraus, 2008; Li et al., 2004; Ruscetti et al., 1998). Thus, PARP1 inhibitors have been developed for cancer treatment, since they would sensitize tumors to radiation and/or chemotherapeutic agents that induce DNA strand breaks. More recently, PARP inhibition has been shown to cause synthetic lethality in homologous recombination deficient tumor cells, such as BRCA1 or BRCA2 deficient cells, by blocking base excision repair and therefore has been used in clinic trials for the treatment of BRCA-deficient tumors (Bryant et al., 2005; 
Farmer et al., 2005; Patel et al., 2011)

PARP2 can also be activated by DNA strand breaks (Ame et al., 1999). PARP1- and PARP2-knockout cells are sensitive to DNA damage, exhibiting marked increased chromatin instability and repair defects (Schreiber et al., 2002). PARP1 and PARP2 knockout mice display similar hypersensitivity to ionizing radiation (de Murcia et al., 2003), suggesting that both PARP1 and PARP2 are involved in DNA damage repair. Likewise, PARP3 is similar to PARP1 and PARP2, since its activity is also stimulated by DNA strand breaks. Recent study suggests that PARP3, and APLF (aprataxin and PNK-like factor) work cooperatively to facilitate double-strand break repair by promoting DNA ligation (Rulten et al., 2011). Therefore, this group of PARPs, PARP $1 / 2 / 3$, is activated by DNA damage and plays critical roles in DNA damage signaling and DNA repair.

PARP1 is the major PARP responding to DNA damage. However, comparing to PARP1 or PARP2 deficient mice, which are sensitive to DNA damage, the PARP1/2 double KO mice are embryonic lethal (de Murcia et al., 2003), indicating that PARP1 and PARP2 at least share some redundant functions in vivo. Whether PARP1/2/3 work together or separately in DNA damage repair remains unknown. In response to DNA damage, many DNA damage repair proteins as well as PARP1 are recruited to DNA damage sites. At the same time, PARP1 catalyzes ADP-ribosylation of its target proteins at sites of DNA damage. Although the connections between ADP-ribosylation and the recruitment for these DNA damage repair proteins have not been fully elucidated, recent studies suggest that a subset of BRCT and FHA domains may have PAR-binding activity and thus participate in damage-induced recruitment of DNA damage checkpoint and repair proteins (Li et al., 2013).

\section{Tankyrases}

Tankyrase1/PARP5a and tankyrase2/PARP5b are closely related, both having long ankyrin repeat regions at the $\mathrm{N}$-terminal region, which are required for protein-protein interaction. They also contain sterile alpha modules in the middle, which is required for homodimerization and heterodimerization, and catalytic PARP domains at the C-terminus (Hsiao and Smith, 2008). The difference between these two proteins is that tankyrase 2 lacks the homopolymeric tracks of the His, Pro, and Ser domain with unknown function, which is present at the N-terminus of tankyrase 1. Otherwise, tankyrase 1 and tankyrase 2 have very similar domain structures and functions (Sbodio et al., 2002).

Tankyrases are known to have diverse functions by targeting different substrates for ADP-ribosylation. Researchers first identified that tankyrases affect telomere extension by PARsylating TRF1, which negatively regulates telomere extension (Smith et al., 1998). Mechanistically, it is known that tankyrases interact with and PARsylate TRF1, promoting the proteasomal degradation of TRF1 and thus leading to its release from telomeres to promote telomere extension (Smith and de Lange, 2000). Tankyrase 1 can also PARsylate nuclear mitotic apparatus protein (NUMA) to control sister chromatid cohesion (Chang et al., 2005). In addition, tankyrase 1 has also been shown to PARsylate Miki to promote prometaphase progression (Ozaki et al., 2012). Moreover, Tankyrase1 can PARsylate 3BP2, leading to its degradation, and thus facilitating normal bone formation (Levaot et al., 2011).

Tankyrases have recently been recognized as potential drug targets for cancer treatment (Riffell et al., 2012). Tankyrases PARsylate and promote the proteasome-dependent degradation of axin, which leads to the activation of the Wnt signaling pathway (Huang et al., 2009). Mechanistically, it is now known that the PAR-binding E3 ligase RNF146 mediates the ubiquitination and degradation of PARsylated axin (Zhang et al., 2011a). Moreover, Zhang and colleagues demonstrated that the tankyrase inhibitor XAV939 could stabilize axin1/2 and thus inhibit the Wnt signaling pathway (Huang et al., 2009). Therefore, tankyrase inhibitors are being actively pursued as possible anticancer agents (Riffell et al., 2012).

\section{CCCH-type PARPs}

The CCCH-type PARPs PARP7, PARP12, and PARP13 have a common Cys-Cys-Cys-His zinc finger domain and common WWE domains. PARP7/TiPARP is a mono-ADP-ribosyltransferase that is regulated by the aryl hydrocarbon receptor (AHR). This CCCH-type PARP co-localizes with the AHR in the nucleus and transcriptionally represses AHR (MacPherson et al., 2013). PARP12 is involved in the regulation of Venezuelan equine encephalitis virus (VEEV) replication (Atasheva et al., 2012). PARP13 is catalytically inactive; but an isoform of PARP13 lacking PARP domain (ZAP) has been shown to interact with viral RNA and prohibit viral RNA accumulation (Gao et al., 2002). Overall, this family of PAPRs has not been extensively studied. However, given the presence of $\mathrm{CCCH}$ Zinc finger domain and the PAR-binding WWE domain on this group of PARPs, it is likely that these PARPs may engage in some interesting PAR-dependent regulations that need to be further elucidated.

\section{Macro-PARPs}

Macro-PARPs are PARPs that contain Macro domains, which include PARP9, PARP14 and PARP15. The macro-PARP BAL1/ PARP9 is a transcription modulator that is differentially expressed in diffuse large B-cell lymphoma cells (Juszczynski et al., 2006). More recently, BAL1/PARP9 and it partner E3 ligase BBAP have been shown to participate in DNA damage response by mediating protein ubiquitination, which is involved in the recruitment of double-strand break repair proteins 53BP1 and BRCA1 (Yan et al., 2013).

BAL2/PARP14 contains three macrodomains and a WWE domain. Similar to PARP9, PARP14 has also been suggested to regulate gene transcription. It interacts with STAT6 and increases STAT6 transcriptional activity (Mehrotra et al., 2011). BAL3/PARP15 contains two macrodomains. It can bind to mono-ADP-ribose or poly-ADP-ribose via its macrodomain and exhibit mono-ADP-ribosylation activity (Venkannagari et al., 2013). However, there is no known function of PARP15.

\section{Other PARPs}

There are a number of PARPs that do not share any common domain structures. These include PARP4, PARP6, PARP8, PARP10, PARP11, and PARP16. PARP4/vPARP is the largest protein in the PARP family and is a component of the vaults, which is $13 \mathrm{MDa}$ ribonucleoprotein complex present in the cytoplasm of cells (Raval-Fernandes et al., 2005). PARP4/vPARPdeficient mice exhibited increased formation of carcinogeninduced tumors (Raval-Fernandes et al., 2005), indicating that PARP4 may have an unknown function in tumor suppression. PARP6 has mono-ADP-ribosylation activity and negatively regulates cellular proliferation (Tuncel et al., 2012). PARP8 and PARP11 also possess mono-ADP-ribosylation activity, but little is known about the functions of these two proteins (Scarpa et al., 2013). PARP10 lacks glutamate residue within its catalytic domain. It has mono-ADP-ribosylation activity but cannot promote poly-ADP-ribosylation (Kleine et al., 2008). This PARP interacts with Myc and inhibits c-Myc- and E1A-mediated trans- 
The PARP family

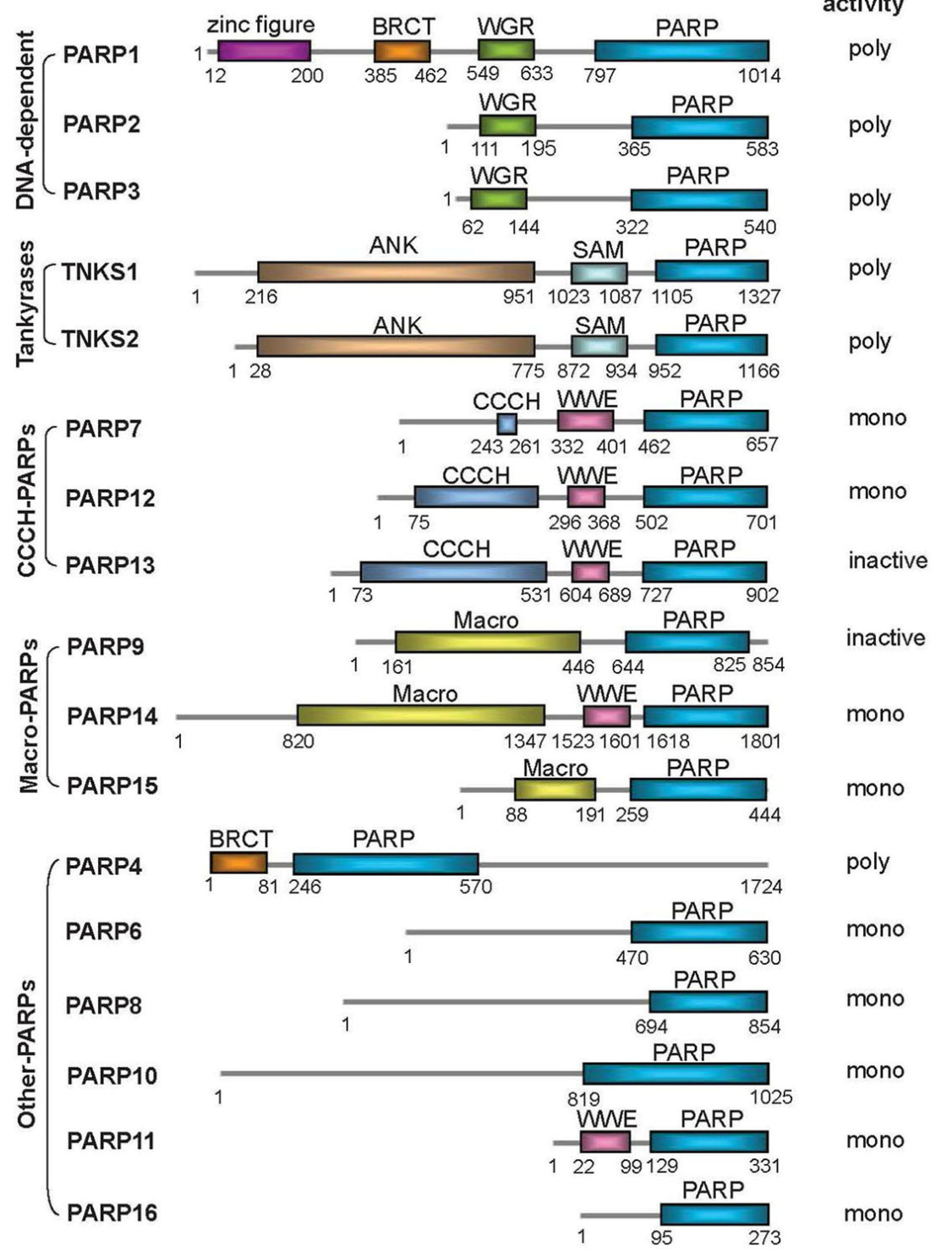

Fig. 1. The domain organization of PARPs. The PARP domain is the catalytic domain and is required for $\mathrm{NAD}^{+}$binding and PAR synthesis. The zinc finger domain is an DNA-binding domain. The BRCA1 $C$ terminus (BRCT), ankyrin repeat (ANK) and sterile $\alpha$-motif (SAM) domains are protein-protein interaction domains. The $\mathrm{CCCH}$ domain is a Cys-CysCys-His zinc finger domain. The WGR is a functionally unknown domain. Macro and WWE are PAR-binding domains. formation (Yu et al., 2005). PARP16 acts as a mono-ADP-ribosyltransferase and negatively regulates the protein level of karyopherin- $\beta 1$ (Di Paola et al., 2012). PARP16 promotes ADPribosylation of PERK and IRE1 $\alpha$ and is required for their activation during endoplasmic reticulum stress (Jwa and Chang, 2012). Overall, these PARPs have not been widely studied and we only have a glimpse of their cellular and physiological functions.

\section{THE PAR RECOGNITION DOMAINS}

The PAR-binding motif

The PAR-binding motif (PBM) is the first identified PAR recognition domain (Pleschke et al., 2000). It is present in most of the known PAR-binding proteins. The consensus PBM sequence is refined to an eight-amino-acid motif: [HKR]-X-X-[AIQVY]-[KR]-
[AILV]-[FILPV] (Gagne et al., 2008). The PBM-containing proteins are involved in diverse cellular processes. For example, both P53 and P21 contain PBM and are key factors in the regulation of cell cycle progression and proliferation, especially in response to stresses like DNA damage (Fahrer et al., 2007). In addition, $\mathrm{x}$-ray repair cross-complementing protein 1 (XRCC1) also has PBM and is rapidly recruited to DNA damage sites, where PARP1-mediated poly-ADP-ribosylation occurs (Pleschke et al., 2000). Apoptosis-inducing factor 1 (AIF1), another PBMcontaining PAR-binding protein, is critically important for PARP1induced cell death (Yu et al., 2006). Thus, many of these proteins are involved in certain aspects of DNA damage response.

The macrodomain

The macrodomain contains $140-190$ residues and was initially 


\section{Macro domain containing proteins}

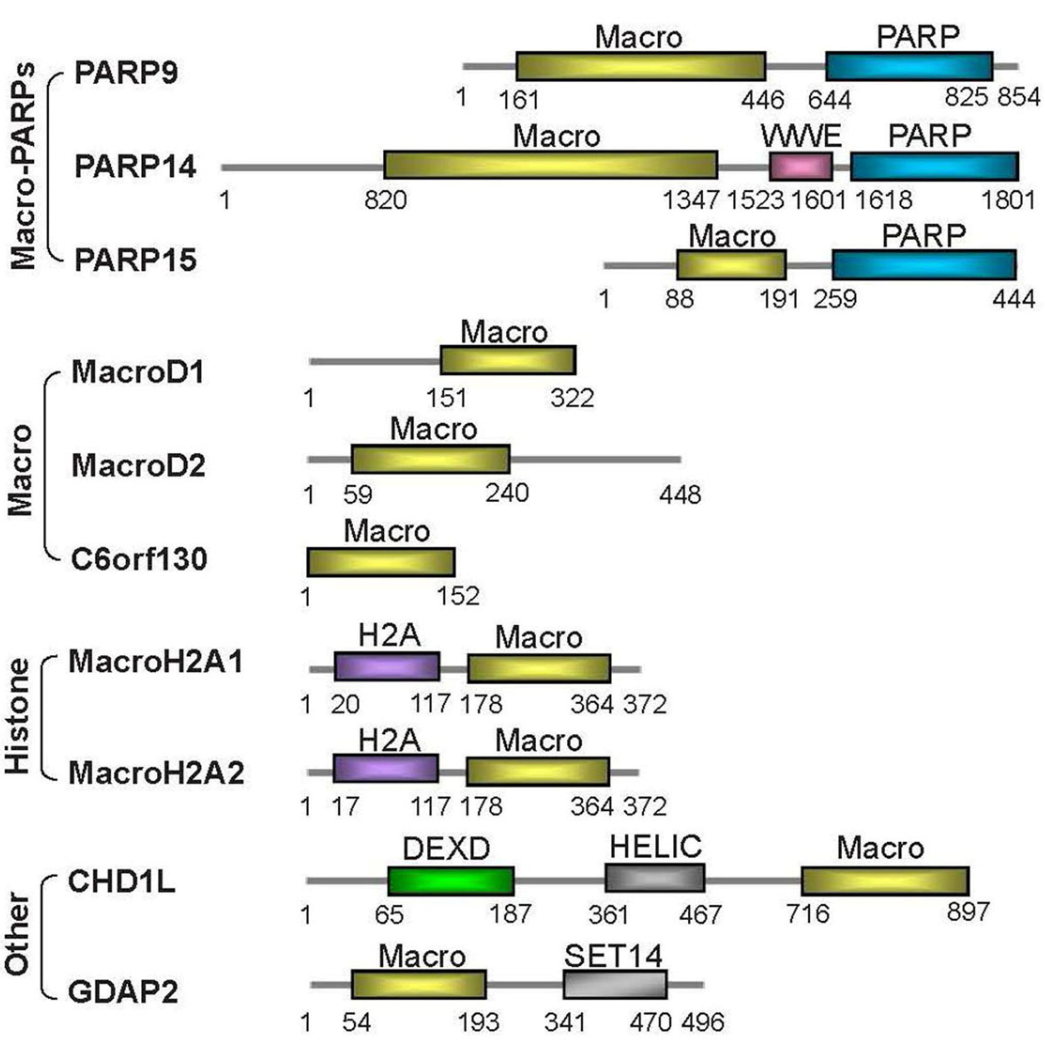

Fig. 2. Schematic diagrams of macro domain-containing proteins. The PARP is the catalytic domain and is required for $\mathrm{NAD}^{+}$ binding and PAR synthesis. Macro and WWE are PAR-binding domains. H2A is the domain in $\mathrm{MacroH} 2 \mathrm{As}$ that shares extensive sequence homolog with histone H2A. DEXD is a DEAD-like helicase domain, which contains ATP-binding region. HELIC is the helicase superfamily c-terminal domain. SEC14 is the Sec14p-like lipid-binding domain. discovered in histone variant macroH2A (Pehrson and Fried, 1992). The macrodomain-containing proteins currently have 10 members and can be categorized into four groups (Chen et al., 2011; Han et al., 2011) (Fig. 2). As discussed above, PARP9, PARP14, and PARP15, which make up the BAL1 family, contain two or three macrodomains. PARP14 and PARP15 exhibit mono-ADP-ribosylation activity while PARP9 seems to be inactive (Aguiar et al., 2005). The MacroD1, MacroD2, and C6orf130 have recently been shown to possess enzymatic activity and participate in the removal of mono-ADP-ribose from substrate proteins (Jankevicius et al., 2013; Rosenthal et al., 2013; Sharifi et al., 2013). Additionally, macroH2A1 and macroH2A2, which are histone subunits, function in transcriptional regulation (Changolkar et al., 2007; Costanzi and Pehrson, 2001). Finally, CHD1L is an ATP-dependent chromatin remodeler (Gottschalk et al., 2009), while GDAP2 is induced by ganglioside synthase expression (Neuvonen and Ahola, 2009). Besides MarcoD1/2 and C6orf130 that have terminal ADP-ribose protein glycohydrolase activity, the functional significance of other macrodomains has not been extensively studied.

\section{PAR-binding zinc finger}

PAR-binding zinc finger (PBZ) is a recently identified zinc finger domain that binds to PAR (Ahel et al., 2008). There are three known PBZ-containing proteins. Checkpoint protein with FHA and RING domains (CHFR) is a tumor suppressor that functions in an early mitotic checkpoint by preventing cells from entering into mitosis (Scolnick and Halazonetis, 2000). APLF is also an FHA domain-containing protein that participates in DNA damage responsive pathway (lles et al., 2007; Rulten et al., 2008). Mutation of PBZ domain in either CHFR or APLF abolishes their ability to bind to PAR (Ahel et al., 2008). Moreover, PBZ domain mutant of CHFR abrogates its function in mitotic checkpoint control (Ahel et al., 2008), but the underlying mechanism remains unclear. Another PBZ-containing protein in mammals is the DNA cross-link repair protein SNM1A (Oberoi et al., 2010).

\section{The WWE domain}

The WWE domain is the most interesting of the recently identified PAR-binding domains, as some WWE-containing proteins exhibit E3 ligase activity and are critical not only for the recognition of PARsylated proteins but also mediating ubiquitination and proteasomal degradation of these modified proteins (Aravind, 2001). The WWE domain-containing proteins can be categorized into three groups (Fig. 3). The first group consists of PARP7, PARP11, PARP12, PARP13, and PARP14, all of which have one or two WWE domains and a PARP domain (Wang et al., 2012). The second group consists of the E3-ubiquitin ligases Deltex1, Deltex2, Deltex4, HUWE1, TRIP12, and RNF146/ Iduna. Deltex1, Deltex2, and Deltex4 are homologs of Drosophila Deltex, which is a critical regulator of Notch signaling (Matsuno et al., 1998). HUWE1 is the E3 ligase for apoptotic protein Mcl1 (Zhong et al., 2005). TRIP12 is thought to regulate the accumulation of RNF168 and downstream DNA damage pathways (Gudjonsson et al., 2012). Of the proteins in this group, 


\section{WWE domain containing proteins}

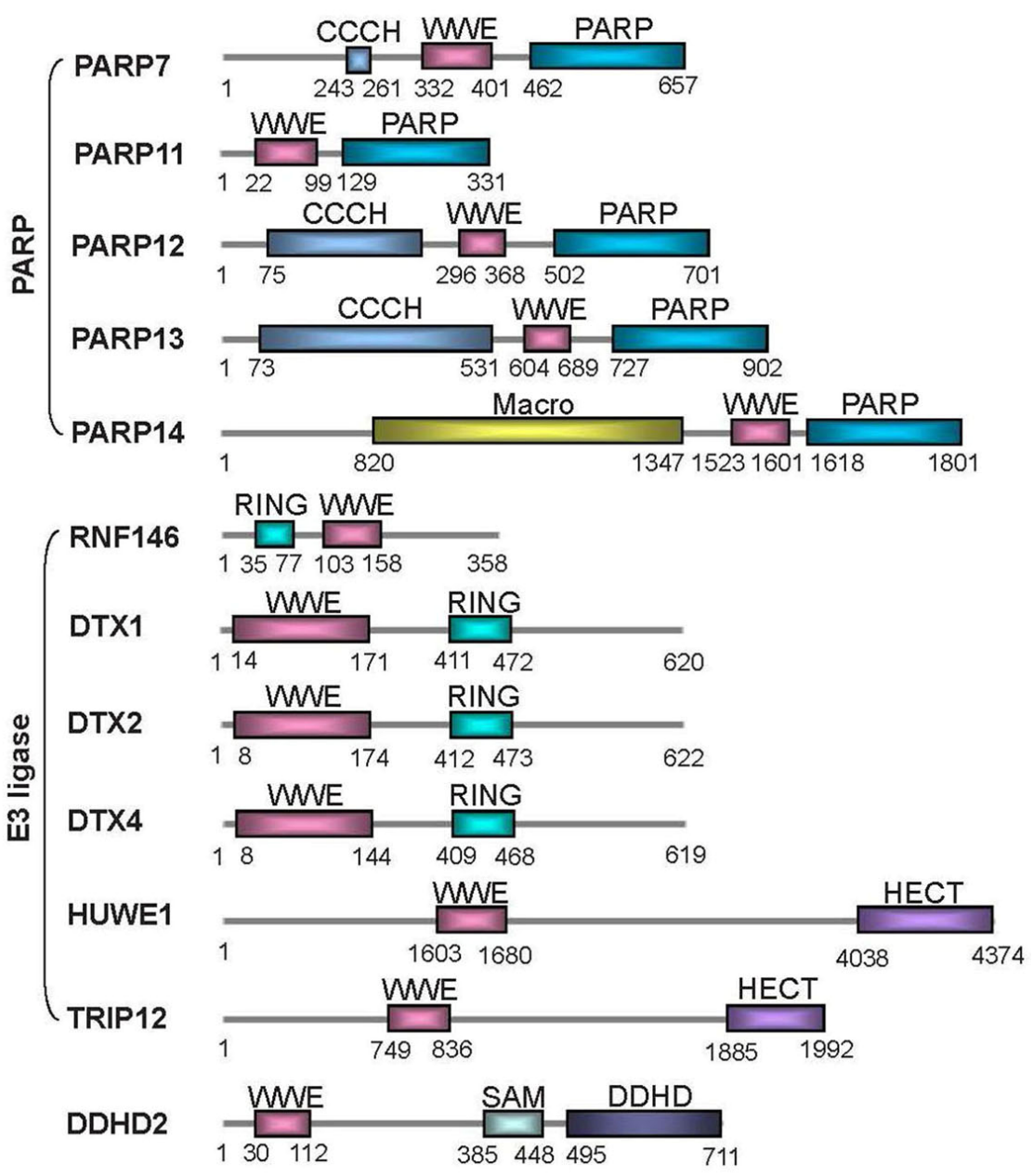

Fig. 3. Schematic diagrams of WWE domain-containing proteins. The PARP is the catalytic domain and is required for $\mathrm{NAD}^{+}$ binding and PAR synthesis. The sterile $\alpha$ motif (SAM) is a protein-protein interaction domain. The $\mathrm{CCCH}$ is a Cys-Cys-Cys-His zinc finger domain. Macro and WWE are PAR-binding domains. The RING domain has E3 ubiquitin ligase activity. HECT domain (homologous to E6-AP carboxyl terminus) also contains E3 ligase activity. DDHD (Asp and His-containing domain) has phospholipase activity. only RNF146/Iduna has exhibited a functional connection between its E3 ligase activity and ADP-ribosylation. RNF146 protects neurons by interfering with PAR polymer-induced cell death (Andrabi et al., 2011). RNF146 also protects against cell death induced by DNA damage via PAR-dependent ubiquitination and proteasomal degradation of PARP1 (Kang et al., 2011). The most exciting finding regarding RNF146 is that it can regulate the WNT signaling pathway by mediating tankyrase-dependent degradation of axin. RNF146 binds directly to ADP-ribosylated axin and promotes axin ubiquitination and degradation (Zhang et al., 2011b). These findings raise the question whether or not other WWE domain-containing E3 ligases also target various PARP substrates for ubiquitination and degradation. Moreover, RNF146 only binds to poly-ADP-ribose, but not mono-ADP-ribose (Wang et al., 2012), indicating that there may be other yet-to-be-identified E3 ligases that recognize monoADP-ribose and mediate proteasome-dependent degradation of mono-ribosylated substrates.

The third group of WWE domain-containing proteins is actually just one protein: DDHD2. This protein has one WWE domain and one DDHD phospholipase domain and is involved in intercellular membrane trafficking at the Golgi complex/endoplasmic reticulum interface (Gonzalez et al., 2013).
BRCT and FHA domains

BRCT and FHA domains are known phospho-peptide binding domains that participate in DNA damage response and cell cycle regulations (Glover et al., 2004; Li et al., 2002; Yu et al., 2003). However, recent studies demonstrated that the FHA domains of APTX and PNKP, and the BRCT domains of BARD1, DNA Ligase 4, XRCC1 and NBS1 recognize Poly-ADP-ribose, and this PAR-binding ability is important for their recruitment to DNA damage sites ( $\mathrm{Li}$ and $\mathrm{Yu}, 2013$; $\mathrm{Li}$ et al., 2013). These studies further expand PAR-binding motifs and highlight the roles of ADP-ribosylation in signaling transduction.

\section{PAR-REMOVING ENZYMES}

Both mono-ADP-ribosylation and PARsylation can be reversed by PAR-removing enzymes. PAR glycohydrolase (PARG) is the best known PAR removing enzyme. It possesses both endoglycohydrolase and exoglycohydrolase activity and is responsible for hydrolysis of ribose-ribose bonds within polyADP-ribose chains (Ikejima and Gill 1988). PARG cleaves polyADP-ribose chains into free chains or mono-ADP-riboses (Lin et al., 1997; Slade et al., 2011). PARG has several isoforms owing to alternative splicing: a very active full-length $110-\mathrm{kDa}$ 


\section{Working model of ADP-ribose cutting enzymes}

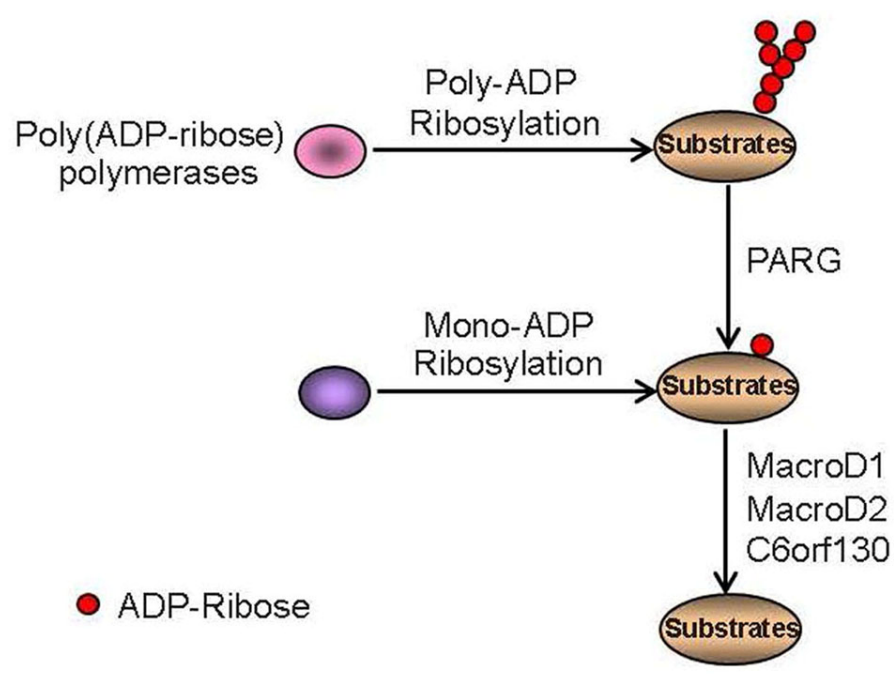

Fig. 4. Working hypothesis of sequential PAR removal by PARG and other ADP-ribose hydrolyases nuclear protein, a shorter $102-\mathrm{kDa}$ nuclear isoform, and a $60-$ $\mathrm{kDa}$ variant located in mitochondria due to the lack of an NLS (nuclear localization signal) at its $\mathrm{N}$-terminal region. This mitochondrial variant of PARG cannot catalyze PAR degradation (Meyer-Ficca et al., 2004). PARG knockout leads to embryonic lethality in mice and PARG-deficient cells are sensitive to genotoxic stress (Koh et al., 2004), demonstrating critical functions of $P A R G$ in genome maintenance.

Another PAR-removing enzyme is the 39-kDa protein ADPribosyl hydrolase 3 , which generates free ADP-ribose from the poly-ADP-ribose chain but cannot cleave the ADP-riboseprotein bond, therefore leading to the formation of mono-ADPribosylated proteins (Moss et al., 1992; Oka et al., 2006). The enzymes that can remove mono-ADP-ribose were unknown for a long time. Recently, several groups reported that some macrodomain-containing proteins, MacroD1, MacroD2, and C6orf130, have ADP-ribose hydrolase activity and can remove monoADP-ribose from substrate proteins by cleaving the riboseprotein bond (Jankevicius et al., 2013; Rosenthal et al., 2013; Sharifi et al., 2013), These findings significantly contributed to the understanding of PAR removal and recycle (Fig. 4).

\section{CONCLUSION}

ADP-ribosylation is one of the protein posttranslational modifications that has been studied for a long time. However, most of the studies focused on PARP1, which is the ubiquitous and best characterized member of the PARP family. Recent studies started to elucidate the biochemical activities and biological functions of other PARPs, which have greatly increased our knowledge of diverse functions of ADP-ribosylation in a variety of cellular processes.

The biology of ADP-ribosylation includes the synthesis, recognition, degradation, and recycling of this important biological moiety. All 17 PARPs are characterized by PARP domain, but they function differently in ADP-ribose synthesis (Scarpa et al., 2013). While PARP1-PARP4 and tankyrase1/2 have poly-ADPribosylation activity, PARP9 and PARP13 are thought to be catalytically inactive, while other PARPs exhibit mono-ADPribosylation activity. Besides the well-studied PARP1, resear- chers made exciting discoveries about the function of tankyrases in targeting axin degradation and activation of the Wnt pathway. However, for most PARPs, their functions, substrates, and how they are regulated are still largely unknown. Furthermore, the PAR recognition domains have now been appreciated as important mediators involved in signal transduction via ADPribosylation. Specifically, the PBM and a subset of FHA and BRCT domains are critically important for the recruitment of checkpoint and repair proteins to DNA damage sites, while the WWE domain of RNF146 is required for E3 ligase-mediated proteasomal degradation of ribosylated proteins. It remains to be determined whether there are new PAR-binding domains and PAR-binding proteins. On the other hand, PARG is the main enzyme that hydrolyzes ribose-ribose bonds. Recent discoveries that macrodomain-containing proteins have the activity to cleave mono-ADP-ribose from substrates provide another piece of the puzzle and underscore the reversible nature of ADP-ribosylation as a signaling moiety. How the activities of PARG and macrodomain-containing proteins are regulated and coordinated is another challenge the field is facing.

\section{ACKNOWLEDGMENTS}

We would like to thank all our colleagues in Dr. Chen's laboratory for insightful discussion. We thank Keithlee Dolinta for editing the manuscript. This work was supported in part by the Department of Defense Era of Hope research scholar award to JC (W81XWH-09-1-0409). J.C. is also a recipient of an Era of Hope Scholar award from the Department of Defense (W81 XWH-05-1-0470) and a member of M.D. Anderson Cancer Center (CA016672).

\section{REFERENCES}

Aguiar, R.C., Takeyama, K., He, C., Kreinbrink, K., and Shipp, M.A. (2005). B-aggressive lymphoma family proteins have unique domains that modulate transcription and exhibit poly(ADP-ribose) polymerase activity. J. Biol. Chem. 280, 33756-33765.

Ahel, I., Ahel, D., Matsusaka, T., Clark, A.J., Pines, J., Boulton, S.J., and West, S.C. (2008). Poly(ADP-ribose)-binding zinc finger motifs in DNA repair/checkpoint proteins. Nature 451, 81-85.

Ame, J.C., Rolli, V., Schreiber, V., Niedergang, C., Apiou, F., Decker, P., Muller, S., Hoger, T., Menissier-de Murcia, J., and de 
Murcia, G. (1999). PARP-2, A novel mammalian DNA damagedependent poly(ADP-ribose) polymerase. J. Biol. Chem. 274, 17860-17868.

Andrabi, S.A., Kang, H.C., Haince, J.F., Lee, Y.I., Zhang, J., Chi, Z., West, A.B., Koehler, R.C., Poirier, G.G., Dawson, T.M., et al. (2011). Iduna protects the brain from glutamate excitotoxicity and stroke by interfering with poly(ADP-ribose) polymer-induced cell death. Nat. Med. 17, 692-699.

Aravind, L. (2001). The WWE domain: a common interaction module in protein ubiquitination and ADP ribosylation. Trends Biochem. Sci. 26, 273-275.

Atasheva, S., Akhrymuk, M., Frolova, E.I., and Frolov, I. (2012). New PARP gene with an anti-alphavirus function. J. Virol. 86, 81478160.

Bryant, H.E., Schultz, N., Thomas, H.D., Parker, K.M., Flower, D., Lopez, E., Kyle, S., Meuth, M., Curtin, N.J., and Helleday, T. (2005). Specific killing of BRCA2-deficient tumours with inhibitors of poly(ADP-ribose) polymerase. Nature 434, 913-917.

Chang, W., Dynek, J.N., and Smith, S. (2005). NuMA is a major acceptor of poly(ADP-ribosyl)ation by tankyrase 1 in mitosis. Biochem. J. 391, 177-184.

Changolkar, L.N., Costanzi, C., Leu, N.A., Chen, D., McLaughlin, K.J., and Pehrson, J.R. (2007). Developmental changes in histone macroH2A1-mediated gene regulation. Mol. Cell. Biol. 27, 2758-2764.

Chen, D., Vollmar, M., Rossi, M.N., Phillips, C., Kraehenbuehl, R., Slade, D., Mehrotra, P.V., von Delft, F., Crosthwaite, S.K., Gileadi, O., et al. (2011). Identification of macrodomain proteins as novel O-acetyl-ADP-ribose deacetylases. J. Biol. Chem. 286, 13261-13271.

Costanzi, C., and Pehrson, J.R. (2001). MACROH2A2, a new member of the MARCOH2A core histone family. J. Biol. Chem. 276, 21776-21784.

de Murcia, J.M.N., Ricoul, M., Tartier, L., Niedergang, C., Huber, A., Dantzer, F., Schreiber, V., Ame, J.C., Dierich, A., LeMeur, M., et al. (2003). Functional interaction between PARP-1 and PARP-2 in chromosome stability and embryonic development in mouse. EMBO J. 22, 2255-2263.

Di Paola, S., Micaroni, M., Di Tullio, G., Buccione, R., and Di Girolamo, M. (2012). PARP16/ARTD15 is a novel endoplasmicreticulum-associated mono-ADP-ribosyltransferase that interacts with, and modifies karyopherin-beta 1. PLoS One 7, e37352.

Fahrer, J., Kranaster, R., Altmeyer, M., Marx, A., and Burkle, A. (2007). Quantitative analysis of the binding affinity of poly(ADPribose) to specific binding proteins as a function of chain length. Nucleic Acids Res. 35, e143.

Farmer, H., McCabe, N., Lord, C.J., Tutt, A.N., Johnson, D.A., Richardson, T.B., Santarosa, M., Dillon, K.J., Hickson, I., Knights, C., et al. (2005). Targeting the DNA repair defect in BRCA mutant cells as a therapeutic strategy. Nature 434, 917-921.

Gagne, J.P., Isabelle, M., Lo, K.S., Bourassa, S., Hendzel, M.J. Dawson, V.L., Dawson, T.M., and Poirier, G.G. (2008). Proteome-wide identification of poly(ADP-ribose) binding proteins and poly(ADP-ribose)-associated protein complexes. Nucleic Acids Res. 36, 6959-6976.

Gao, G., Guo, X., and Goff, S.P. (2002). Inhibition of retroviral RNA production by ZAP, a CCCH-type zinc finger protein. Science 297, 1703-1706

Gibson, B.A., and Kraus, W.L. (2012). New insights into the molecular and cellular functions of poly(ADP-ribose) and PARPs. Nat Rev. Mol. Cell Biol. 13, 411-424.

Glover, J.N., Williams, R.S., and Lee, M.S. (2004). Interactions between BRCT repeats and phosphoproteins: tangled up in two. Trends Biochem. Sci. 29, 579-585.

Gonzalez, M., Nampoothiri, S., Kornblum, C., Oteyza, A.C., Walter, J., Konidari, I., Hulme, W., Speziani, F., Schols, L., Zuchner, S., et al. (2013). Mutations in phospholipase DDHD2 cause autosomal recessive hereditary spastic paraplegia (SPG54). Eur. J. Hum. Genet. [Epub ahead of print]

Gottschalk, A.J., Timinszky, G., Kong, S.E., Jin, J., Cai, Y., Swanson, S.K., Washburn, M.P., Florens, L., Ladurner, A.G., Conaway, J.W., et al. (2009). Poly(ADP-ribosyl)ation directs recruitment and activation of an ATP-dependent chromatin remodeler. Proc. Natl. Acad. Sci. USA 106, 13770-13774.

Gudjonsson, T., Altmeyer, M., Savic, V., Toledo, L., Dinant, C., Grofte, M., Bartkova, J., Poulsen, M., Oka, Y., Bekker-Jensen, S., et al.
(2012). TRIP12 and UBR5 suppress spreading of chromatin ubiquitylation at damaged chromosomes. Cell 150, 697-709.

Han, W., Li, X., and Fu, X. (2011). The macro domain protein family: structure, functions, and their potential therapeutic implications. Mutat. Res. 727, 86-103.

Hassa, P.O., and Hottiger, M.O. (2008). The diverse biological roles of mammalian PARPS, a small but powerful family of poly-ADPribose polymerases. Front Biosci. 13, 3046-3082.

Hsiao, S.J., and Smith, S. (2008). Tankyrase function at telomeres, spindle poles, and beyond. Biochimie 90, 83-92.

Huang, S.M., Mishina, Y.M., Liu, S., Cheung, A., Stegmeier, F., Michaud, G.A., Charlat, O., Wiellette, E., Zhang, Y., Wiessner, S., et al. (2009). Tankyrase inhibition stabilizes axin and antagonizes Wnt signalling. Nature 461, 614-620.

Ikejima, M., and Gill, D.M. (1988). Poly(ADP-ribose) degradation by glycohydrolase starts with an endonucleolytic incision. J. Biol. Chem. 263, 11037-11040.

Iles, N., Rulten, S., El-Khamisy, S.F., and Caldecott, K.W. (2007). APLF (C2orf13) is a novel human protein involved in the cellular response to chromosomal DNA strand breaks. Mol. Cell. Biol. 27, 3793-3803.

Jankevicius, G., Hassler, M., Golia, B., Rybin, V., Zacharias, M. Timinszky, G., and Ladurner, A.G. (2013). A family of macrodomain proteins reverses cellular mono-ADP-ribosylation. Nat. Struct. Mol. Biol. 20, 508-514.

Juszczynski, P., Kutok, J.L., Li, C., Mitra, J., Aguiar, R.C., and Shipp, M.A. (2006). BAL1 and BBAP are regulated by a gamma interferon-responsive bidirectional promoter and are overexpressed in diffuse large B-cell lymphomas with a prominent inflammatory infiltrate. Mol. Cell. Biol. 26, 5348-5359.

Jwa, M., and Chang, P. (2012). PARP16 is a tail-anchored endoplasmic reticulum protein required for the PERK- and IRE1 alphamediated unfolded protein response. Nat. Cell Biol. 14, 1223-1230.

Kang, H.C., Lee, Y.I., Shin, J.H., Andrabi, S.A., Chi, Z.K., Gagne, J.P., Lee, Y.J., Ko, H.S., Lee, B.D., Poirier, G.G., et al. (2011) Iduna is a poly(ADP-ribose) (PAR)-dependent E3 ubiquitin ligase that regulates DNA damage. Proc. Natl. Acad. Sci. USA 108, 14103-14108.

Kleine, H., Poreba, E., Lesniewicz, K., Hassa, P.O., Hottiger, M.O., Litchfield, D.W., Shilton, B.H., and Luscher, B. (2008). Substrate-assisted catalysis by PARP10 limits its activity to monoADP-ribosylation. Mol. Cell 32, 57-69.

Koh, D.W., Lawler, A.M., Poitras, M.F., Sasaki, M., Wattler, S., Nehls, M.C., Stoger, T., Poirier, G.G., Dawson, V.L., and Dawson, T.M (2004). Failure to degrade poly(ADP-ribose) causes increased sensitivity to cytotoxicity and early embryonic lethality. Proc. Natl. Acad. Sci. USA 101, 17699-17704.

Kraus, W.L. (2008). Transcriptional control by PARP-1: chromatin modulation, enhancer-binding, coregulation, and insulation. Curr Opin. Cell Biol. 20, 294-302.

Levaot, N., Voytyuk, O., Dimitriou, I., Sircoulomb, F., Chandrakumar A., Deckert, M., Krzyzanowski, P.M., Scotter, A., Gu, S., Janmohamed, S., et al. (2011). Loss of Tankyrase-mediated destruction of 3BP2 is the underlying pathogenic mechanism of cherubism. Cell 147, 1324-1339.

$\mathrm{Li}, \mathrm{M}$., and Yu, X. (2013). Function of BRCA1 in the DNA damage response is mediated by ADP-ribosylation. Cancer Cell 23, 693704

Li, J., Williams, B.L., Haire, L.F., Goldberg, M., Wilker, E., Durocher, D., Yaffe, M.B., Jackson, S.P., and Smerdon, S.J. (2002). Structural and functional versatility of the FHA domain in DNA-damage signaling by the tumor suppressor kinase Chk2. Mol. Cell 9, 1045-1054

Li, B., Navarro, S., Kasahara, N., and Comai, L. (2004). Identification and biochemical characterization of a Werner's syndrome protein complex with Ku70/80 and poly(ADP-ribose) polymerase-1. J. Biol. Chem. 279, 13659-13667.

Li, M., Lu, L.Y., Yang, C.Y., Wang, S., and Yu, X. (2013). The FHA and BRCT domains recognize ADP-ribosylation during DNA damage response. Genes Dev. 27, 1752-1768.

Lin, W., Ame, J.C., Aboul-Ela, N., Jacobson, E.L., and Jacobson, M.K. (1997). Isolation and characterization of the cDNA encoding bovine poly(ADP-ribose) glycohydrolase. J. Biol. Chem. 272, 11895-11901.

MacPherson, L., Tamblyn, L., Rajendra, S., Bralha, F., McPherson, J.P., and Matthews, J. (2013). 2,3,7,8-Tetrachlorodibenzo-p-dioxin 
poly(ADP-ribose) polymerase (TiPARP, ARTD14) is a monoADP-ribosyltransferase and repressor of aryl hydrocarbon receptor transactivation. Nucleic Acids Res. 41, 1604-1621.

Matsuno, K., Eastman, D., Mitsiades, T., Quinn, A.M., Carcanciu, M.L., Ordentlich, P., Kadesch, T., and Artavanis-Tsakonas, S. (1998). Human deltex is a conserved regulator of Notch signalling. Nat. Genet. 19, 74-78.

Mehrotra, P., Riley, J.P., Patel, R., Li, F., Voss, L., and Goenka, S. (2011). PARP-14 functions as a transcriptional switch for Stat6dependent gene activation. J. Biol. Chem. 286, 1767-1776.

Meyer-Ficca, M.L., Meyer, R.G., Coyle, D.L., Jacobson, E.L., and Jacobson, M.K. (2004). Human poly(ADP-ribose) glycohydrolase is expressed in alternative splice variants yielding isoforms that localize to different cell compartments. Exp. Cell Res. 297, 521-532.

Moss, J., Stanley, S.J., Nightingale, M.S., Murtagh, J.J., Jr., Monaco, L., Mishima, K., Chen, H.C., Williamson, K.C., and Tsai, S.C. (1992). Molecular and immunological characterization of ADPribosylarginine hydrolases. J. Biol. Chem. 267, 10481-10488.

Neuvonen, M., and Ahola, T. (2009). Differential activities of cellular and viral macro domain proteins in binding of ADP-ribose metabolites. J. Mol. Biol. 385, 212-225.

Oberoi, J., Richards, M.W., Crumpler, S., Brown, N., Blagg, J., and Bayliss, R. (2010). Structural basis of poly(ADP-ribose) recognition by the multizinc binding domain of checkpoint with forkheadassociated and RING Domains (CHFR). J. Biol. Chem. 285, 39348-39358.

Oka, S., Kato, J., and Moss, J. (2006). Identification and characterization of a mammalian 39-kDa poly(ADP-ribose) glycohydrolase. J. Biol. Chem. 281, 705-713.

Ozaki, Y., Matsui, H., Asou, H., Nagamachi, A., Aki, D., Honda, H., Yasunaga, S., Takihara, Y., Yamamoto, T., Izumi, S., et al. (2012). Poly-ADP ribosylation of Miki by tankyrase-1 promotes centrosome maturation. Mol. Cell 47, 694-706.

Parsons, J.L., Dianova, II, Allinson, S.L., and Dianov, G.L. (2005). Poly(ADP-ribose) polymerase-1 protects excessive DNA strand breaks from deterioration during repair in human cell extracts. FEBS J. 272, 2012-2021.

Patel, A.G., Sarkaria, J.N., and Kaufmann, S.H. (2011). Nonhomologous end joining drives poly(ADP-ribose) polymerase (PARP) inhibitor lethality in homologous recombination-deficient cells. Proc. Natl. Acad. Sci. USA 108, 3406-3411.

Pehrson, J.R and Fried, V. A. (1992). MacroH2A a core histone containing a large nonhistone region. Science 257, 1398-1400.

Pleschke, J.M., Kleczkowska, H.E., Strohm, M., and Althaus, F.R (2000). Poly(ADP-ribose) binds to specific domains in DNA damage checkpoint proteins. J. Biol. Chem. 275, 40974-40980.

Raval-Fernandes, S., Kickhoefer, V.A., Kitchen, C., and Rome, L.H. (2005). Increased susceptibility of vault poly(ADP-ribose) polymerase-deficient mice to carcinogen-induced tumorigenesis. Cancer Res. 65, 8846-8852.

Riffell, J.L., Lord, C.J., and Ashworth, A. (2012). Tankyrase-targeted therapeutics: expanding opportunities in the PARP family. Nat Rev. Drug Discov. 11, 923-936.

Rosenthal, F., Feijs, K.L.H., Frugier, E., Bonalli, M., Forst, A.H., Imhof, R., Winkler, H.C., Fischer, D., Caflisch, A., Hassa, P.O., et al. (2013). Macrodomain-containing proteins are new monoADP-ribosylhydrolases. Nat. Struct. Mol. Biol. 20, 502-507.

Rouleau, M., Patel, A., Hendzel, M.J., Kaufmann, S.H., and Poirier, G.G. (2010). PARP inhibition: PARP1 and beyond. Nat. Rev. Cancer 10, 293-301.

Rulten, S.L., Cortes-Ledesma, F., Guo, L., Iles, N.J., and Caldecott, K.W. (2008). APLF (C2orf13) is a novel component of poly(ADPribose) signaling in mammalian cells. Mol. Cell. Biol. 28, 46204628.

Rulten, S.L., Fisher, A.E., Robert, I., Zuma, M.C., Rouleau, M., Ju, L., Poirier, G., Reina-San-Martin, B., and Caldecott, K.W. (2011) PARP-3 and APLF function together to accelerate nonhomologous end-joining. Mol. Cell 41, 33-45.

Ruscetti, T., Lehnert, B.E., Halbrook, J., Le Trong, H., Hoekstra, M.F., Chen, D.J., and Peterson, S.R. (1998). Stimulation of the DNA-dependent protein kinase by poly(ADP-ribose) polymerase. J. Biol. Chem. 273, 14461-14467.
Sbodio, J.I., Lodish, H.F., and Chi, N.W. (2002). Tankyrase-2 oligomerizes with tankyrase-1 and binds to both TRF1 (telomererepeat-binding factor 1) and IRAP (insulin-responsive aminopeptidase). Biochem. J. 361, 451-459.

Scarpa, E.S., Fabrizio, G., and Di Girolamo, M. (2013). A role of intracellular mono-ADP-ribosylation in cancer biology. FEBS J. 280, 3551-3562.

Schreiber, V., Ame, J.C., Dolle, P., Schultz, I., Rinaldi, B., Fraulob, V., Menissier-de Murcia, J., and de Murcia, G. (2002). Poly(ADPribose) polymerase-2 (PARP-2) is required for efficient base excision DNA repair in association with PARP-1 and XRCC1. J. Biol. Chem. 277, 23028-23036.

Schreiber, V., Dantzer, F., Ame, J.C., and de Murcia, G. (2006) Poly(ADP-ribose): novel functions for an old molecule. Nat. Rev. Mol. Cell Biol. 7, 517-528.

Scolnick, D.M., and Halazonetis, T.D. (2000). Chfr defines a mitotic stress checkpoint that delays entry into metaphase. Nature 406 430-435.

Sharifi, R., Morra, R., Appel, C.D., Tallis, M., Chioza, B., Jankevicius, G., Simpson, M.A., Matic, I., Ozkan, E., Golia, B., et al. (2013). Deficiency of terminal ADP-ribose protein glycohydrolase TARG1/ C6orf130 in neurodegenerative disease. EMBO J. 32, 1225-1237.

Slade, D., Dunstan, M.S., Barkauskaite, E., Weston, R., Lafite, P., Dixon, N., Ahel, M., Leys, D., and Ahel, I. (2011). The structure and catalytic mechanism of a poly(ADP-ribose) glycohydrolase. Nature 477, 616-620.

Smith, S., and de Lange, T. (2000). Tankyrase promotes telomere elongation in human cells. Curr. Biol. 10, 1299-1302.

Smith, S., Giriat, I., Schmitt, A., and de Lange, T. (1998). Tankyrase, a poly(ADP-ribose) polymerase at human telomeres. Science 282, $1484-1487$

Tuncel, H., Tanaka, S., Oka, S., Nakai, S., Fukutomi, R., Okamoto, M., Ota, T., Kaneko, H., Tatsuka, M., and Shimamoto, F. (2012). PARP6, a mono(ADP-ribosyl) transferase and a negative regulator of cell proliferation, is involved in colorectal cancer development. Int. J. Oncol. 41, 2079-2086.

Venkannagari, H., Fallarero, A., Feijs, K.L.H., Luscher, B., and Lehtio, L. (2013). Activity-based assay for human mono-ADPribosyltransferases ARTD7/PARP15 and ARTD10/PARP10 aimed at screening and profiling inhibitors. Eur. J. Pharm. Sci. 49, 148-156.

Wang, Z., Michaud, G.A., Cheng, Z., Zhang, Y., Hinds, T.R., Fan, E., Cong, F., and Xu, W. (2012). Recognition of the iso-ADPribose moiety in poly(ADP-ribose) by WWE domains suggests a general mechanism for poly(ADP-ribosyl)ation-dependent ubiquitination. Genes Dev. 26, 235-240.

Yan, Q., Xu, R., Zhu, L., Cheng, X., Wang, Z., Manis, J., and Shipp, M.A. (2013). BAL1 and its partner E3 ligase, BBAP, link Poly(ADPribose) activation, ubiquitylation, and double-strand DNA repair independent of ATM, MDC1, and RNF8. Mol. Cell. Biol. 33, 845857

Yu, X., Chini, C.C., He, M., Mer, G., and Chen, J. (2003). The BRCT domain is a phospho-protein binding domain. Science 302, 639642.

Yu, M., Schreek, S., Cerni, C., Schamberger, C., Lesniewicz, K. Poreba, E., Vervoorts, J., Walsemann, G., Grotzinger, J., Kremmer, E., et al. (2005). PARP-10, a novel Myc-interacting protein with poly(ADP-ribose) polymerase activity, inhibits transformation. Oncogene 24, 1982-1993.

Yu, S.W., Andrabi, S.A., Wang, H., Kim, N.S., Poirier, G.G., Dawson, T.M., and Dawson, V.L. (2006). Apoptosis-inducing factor mediates poly(ADP-ribose) (PAR) polymer-induced cell death Proc. Natl. Acad. Sci. USA 103, 18314-18319.

Zhang, Y., Liu, S., Mickanin, C., Feng, Y., Charlat, O., Michaud, G.A., Schirle, M., Shi, X., Hild, M., Bauer, A., et al. (2011a). RNF146 is a poly(ADP-ribose)-directed E3 ligase that regulates axin degradation and Wnt signalling. Nat. Cell Biol. 13, 623-629.

Zhang, Y., Liu, S.M., Mickanin, C., Feng, Y., Charlat, O., Michaud, G.A., Schirle, M., Shi, X.Y., Hild, M., Bauer, A., et al. (2011b) RNF146 is a poly(ADP-ribose)-directed E3 ligase that regulates axin degradation and Wnt signalling. Nat. Cell Biol. 13,623-U292.

Zhong, Q., Gao, W., Du, F., and Wang, X. (2005). Mule/ARF-BP1, a BH3-only E3 ubiquitin ligase, catalyzes the polyubiquitination of $\mathrm{Mcl}-1$ and regulates apoptosis. Cell 121, 1085-1095. 